\title{
Theoretical computer science and discrete mathematics
}

\author{
Ken-ichi KAWARABAYASHI ${ }^{1}$, Kunihiko SADAKANE ${ }^{2}$, and Takeaki UNO ${ }^{3}$ \\ 1,2,3 National Institute of Informatics
}

Recent informatics such as large scale data analysis needs efficient computation. The developments in theoretical computer science give much advance in this task. These divisions and subsets include analysis of algorithms and formal semantics of programming languages. In order to deal with large scale data analysis, we need some mathematical tools from Discrete Mathematics.

Theoretical Computer Science is one of the most vibrant and active areas of scientific study today. Starting half a century ago, even before computers existed, theoretical computer scientists set out to define mathematically the concept of "computation", and to study its power and limits. It is a division or subset of general computer science and mathematics which focuses on more abstract or mathematical aspects of computing. These divisions and subsets include analysis of algorithms and formal semantics of programming languages.

Discrete mathematics is the study of mathematical structures that are fundamentally discrete rather than continuous. In contrast to real numbers that have the property of varying "smoothly", the objects studied in discrete mathematics such as integers and graphs do not vary smoothly in this way, but have distinct, separated values. It has been characterized as the branch of mathematics.

Dealing with discrete objects, questions from theoretical computer science inspired much interest in the combinatorics community, and for many of its leaders became a primary scientific goal. A typical goal is the $P$ versus NP problem, which characterizes difficulties of various problems, and much research has been devoted to analyze complexities of the problems. This collaboration has been extremely beneficial to both the discrete math and theoretical computer science communities, with wealthy exchange of ideas, problems and techniques.

The aim of this special issue is to provide a forum for presenting current research and discussing future research directions in Theoretical Computer Science and Discrete Mathematics for developing academically challenging.

In response to Call For Papers for this special issue, we have received 9 papers, and we have accepted 6 papers out of them. The decisions on selecting papers are based on originality, technical contribution, practical contribution and relevance. In addition to the editors, at least two referees are assigned to each submitted paper. The submissions are thus formally refereed, and they are expected to be in the final format, and will not be published elsewhere.

Let us mention topics in this special issue. They include

1. Sorting algorithm.

2. Well quasi-orderings and better quasi-orderings.

3 . Entropy estimation with suffix arrays.

4. Clique enumeration algorithm.

5. Dynamic programming algorithm.

6. Minor and Immersion.

All the topics mentioned above are very relevant to Discrete Mathematics and Theoretical Computer Science.

We would like to thank all the authors who submitted papers for consideration. We also express our thanks to all the referees who dedicated to a significant amount of time and helped to improve the quality of the papers. Finally, we thank the stuff of the journal office, in particular, Ms Ayumi Shimizu, for their support throughout the editorial process. 


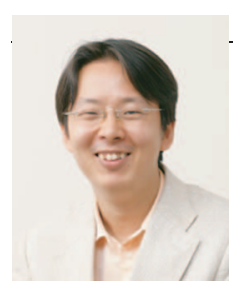
journals, including Siam J. Discrete Mathematics and J. Graph Theory. He has won several domestic prizes, including IBM Science Prize in Computer Science in 2008.

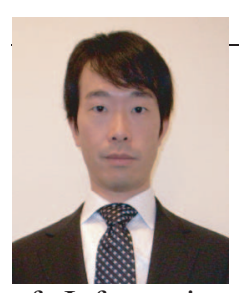

\section{Kunihiko SADAKANE}

Kunihiko SADAKANE received B.S., M.S., and Ph.D. degrees from Department of Information Science, University of Tokyo in 1995, 1997 and 2000 , respectively. He was a research associate at Graduate School of Information Sciences, Tohoku University from 2000 to 2003, and an associate professor at Faculty of Information Science and Electrical Engineering, Kyushu University from 2003 to 2009. Since 2009, he has been an associate professor at National Institute of Informatics. His research interest includes information retrieval, data structures, and data compression. He is a member of IPSJ and IEICE.

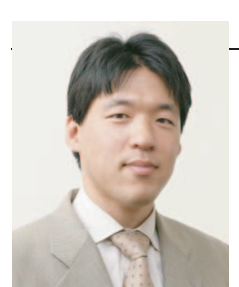

Takeaki UNO

Takeaki UNO received the $\mathrm{PhD}$ degree (Doctor of Science) from Department of Systems Science, Tokyo Institute of Technology Japan, 1998. He was an assistant professor in Department of Industrial and Management Science in Tokyo Institute of Technology from 1998 to 2001, and have been an associate professor of National Institute of Informatics Japan, from 2001. His research topic is discrete algorithms, especially enumeration algorithms, algorithms on graph classes, and data mining algorithms. In data mining area, his main research is on pattern mining algorithms. He got Young Scientists' Prize, of The Commendation for Science and Technology in Japan, in 2010. 